Chaos, Solitons and Fractals, Vol. 37, № 3, 770-780 (2008)

\title{
Application of He's homotopy perturbation method to conservative truly nonlinear oscillators
}

\author{
A. Beléndez, T. Beléndez, A. Márquez and C. Neipp \\ Departamento de Física, Ingeniería de Sistemas y Teoría de la Señal. \\ Universidad de Alicante. \\ Apartado 99. E-03080 Alicante. SPAIN \\ E-mail: a.belendez@ua.es
}

Corresponding author: A. Beléndez

Phone: +34-6-5903651

Fax: +34-6-5903464 


\begin{abstract}
We apply He's homotopy perturbation method to find improved approximate solutions to conservative truly nonlinear oscillators. This approach gives us not only a truly periodic solution but also the period of the motion as a function of the amplitude of oscillation. We find that this method works very well for the whole range of parameters in the case of the cubic oscillator, and excellent agreement of the approximate frequencies with the exact one has been demonstrated and discussed. For the second order approximation we have shown that the relative error in the analytical approximate frequency is approximately $0.03 \%$ for any parameter values involved. We also compared the analytical approximate solutions and the Fourier series expansion of the exact solution. This has allowed us to compare the coefficients for the different harmonic terms in these solutions. The most significant features of this method are its simplicity and its excellent accuracy for the whole range of oscillation amplitude values and the results reveal that this technique is very effective and convenient for solving conservative truly nonlinear oscillatory systems.
\end{abstract}

Keywords: Truly nonlinear oscillators; Approximate solutions; Homotopy perturbation method. 


\section{Introduction}

The study of nonlinear problems is of crucial importance not only in all areas of physics but also in engineering and other disciplines, since most phenomena in our world are essentially nonlinear $[1,2]$ and are described by nonlinear equations. It is very difficult to solve nonlinear problems and, in general, it is often more difficult to get an analytic approximation than a numerical one for a given nonlinear problem [2]. There are several methods used to find approximate solutions to nonlinear problems [3], such as perturbation techniques [4-14] or harmonic balance based methods [15-22]. An excellent review on some asymptotic methods for strongly nonlinear equations can be found in detail in Ref. [23]. In general, given the nature of a nonlinear phenomenon, the approximate methods can only be applied within certain ranges of the physical parameters and/or to certain classes of problems [11].

In this paper, we will show how to solve conservative truly nonlinear oscillators [24] by using the homotopy perturbation method [23], which yields a very rapid convergence of the solution series. This method requires neither a linear term nor a perturbation parameter, and if this parameter exists, the method does not require the parameter to be small. This perturbation approach has been applied not only to nonlinear oscillators [25-27] but also to other nonlinear problems [28-37].

In section 2 we briefly review the alternative Lindstedt-Poincaré perturbation procedure $[8,13]$. In section 3 we outline He's homotopy perturbation method for conservative truly nonlinear oscillators. In section 4 we illustrate the applicability of this method by means an illustrative example of the simplest conservative truly nonlinear oscillator. In section 5 we compare the approximate frequencies and periods of the motion and approximate solutions obtained with the approximate periods and solutions constructed using the harmonic balance method, as well as with the exact frequency and solution for the example analyzed. For the cubic oscillator we will see that only two iterations leads to high accuracy of the solution, providing an effective and convenient mathematical tool for this kind of nonlinear oscillators. We also compare the analytical approximate solutions and the Fourier series expansion of the exact solution. This allows us to compare the coefficients for the different harmonic terms in these solutions. 
Finally, we present our conclusions in section 6 and an appendix contains the exact solution for the conservative truly nonlinear oscillator analyzed in this paper.

\section{Alternative Lindstedt-Poincaré method}

We consider a nonlinear oscillator governed by the following dimensionless nonlinear differential equation

$$
\frac{\mathrm{d}^{2} x(t)}{\mathrm{d} t^{2}}+x(t)+\varepsilon g(x(t))=0
$$

with initial conditions

$$
x(0)=A \text { and } \quad \frac{\mathrm{d} x}{\mathrm{~d} t}(0)=0
$$

In Eq. (1) $F(x)=-x-\varepsilon g(x)$ is the dimensionless restoring force and $g(x)$ is a nonlinear function. For this oscillatory system the restoring force, $F(x)$, has two terms, the first term, $-x$, is a linear term, while the second term, $-\varepsilon g(x)$, is a nonlinear term, where $\varepsilon$ is a positive parameter. Eq. (1) corresponds to a conservative system and its "potential function" is

$$
V(x)=\frac{1}{2} x^{2}+\varepsilon \int g(x) \mathrm{d} x
$$

and $E$ is the "total energy" of the nonlinear oscillator. This system oscillates with an unknown frequency $\omega$ (thus a period $T=2 \pi / \omega$ ). In Eq. (1) the nonlinear term $\varepsilon g(x)$ is treated as a perturbation to the equation of motion of an harmonic oscillator and $\varepsilon$ is the perturbation parameter. The angular frequency for the linear oscillator (for $\varepsilon=0$ ) is 1 . This alternative Lindstedt-Poincaré perturbation approach resembles the standard Lindstedt-Poincare method and is based on the expansion of the solution $x(t)$ and $\omega^{2}$ in powers of $\varepsilon$ [8]

$$
x(t)=x_{0}(t)+\varepsilon x_{1}(t)+\varepsilon^{2} x_{2}(t)+\ldots
$$




$$
\omega^{2}=1+\varepsilon \omega_{1}+\varepsilon^{2} \omega_{2}+\ldots
$$

In Eq. (6) $\omega$ is the unknown frequency and the first term of the series expansion, 1, is the square of the frequency of the harmonic oscillator (for $\varepsilon=0$ ), i.e., the factor that multiplies the linear term, $x(t)$ in Eq. (1). It is possible to rewrite Eq. (6) as follows

$$
1=\omega^{2}-\varepsilon \omega_{1}-\varepsilon^{2} \omega_{2}-\ldots
$$

The alternative Lindstedt-Poincaré perturbation procedure is based on the substitution of Eqs. (5) and (7) into Eq. (1). This substitution gives

$$
\begin{aligned}
& \left(x_{0}^{\prime \prime}+\varepsilon x_{1}^{\prime \prime}+\varepsilon^{2} x_{2}^{\prime \prime}+\ldots\right)+\left(\omega^{2}-\varepsilon \omega_{1}-\varepsilon^{2} \omega_{2}-\ldots\right)\left(x_{0}+\varepsilon x_{1}+\varepsilon^{2} x_{2}^{\prime \prime}+\ldots\right) \\
& \quad+\varepsilon g\left(x_{0}+\varepsilon x_{1}+\varepsilon^{2} x_{2}^{\prime \prime}+\ldots\right)=0
\end{aligned}
$$

where primes denote differentiation with respect to $t$. Setting the coefficients of the various powers of $\varepsilon$ to zero leads to the following system of linear differential equations

$$
\begin{aligned}
& x_{0}^{\prime \prime}+\omega^{2} x_{0}=0 \\
& x_{1}^{\prime \prime}+\omega^{2} x_{1}=\omega_{1} x_{0}-g\left(x_{0}\right) \\
& x_{2}^{\prime \prime}+\omega^{2} x_{2}=\omega_{2} x_{0}+\omega_{1} x_{1}-x_{1} g^{\prime}\left(x_{0}\right) \\
& x_{3}^{\prime \prime}+\omega^{2} x_{3}=\omega_{3} x_{0}+\omega_{2} x_{1}+\omega_{1} x_{2}-x_{2} g^{\prime}\left(x_{0}\right)-\frac{1}{2} x_{1}^{2} g^{\prime \prime}\left(x_{0}\right)
\end{aligned}
$$

The initial conditions are replaced by

$$
x_{0}(0)=A, \quad x_{0}^{\prime}(0)=0, \quad x_{j}(0)=0, \quad x_{j}^{\prime}(0)=0, \quad j \geq 1
$$

In Eqs. (8)-(12) we have taken into account the following expression [10] 


$$
\begin{gathered}
g(x)=g\left(x_{0}+\varepsilon x_{1}+\varepsilon^{2} x_{2}+\ldots\right) \approx g\left(x_{0}\right)+\varepsilon x_{1} g^{\prime}\left(x_{0}\right)+\varepsilon^{2}\left[x_{2} g^{\prime}\left(x_{0}\right)+\frac{1}{2} x_{1}^{2} g^{\prime \prime}\left(x_{0}\right)\right] \\
+\varepsilon^{3}\left[x_{3} g^{\prime}\left(x_{0}\right)+x_{1} x_{2} g^{\prime \prime}\left(x_{0}\right)+\frac{1}{6} g^{\prime \prime \prime}\left(x_{0}\right)\right]+O\left(\varepsilon^{4}\right)
\end{gathered}
$$

In Eqs. (11) and (13) we choose the value of the coefficients $\omega_{n}$ in order to remove any secular term from the perturbation equation of order $n$.

\section{He's homotopy perturbation method}

We consider the following more general dimensionless equation of motion of a nonlinear oscillator

$$
\frac{\mathrm{d}^{2} x(t)}{\mathrm{d} t^{2}}+f(x(t))=0
$$

with the initial conditions in Eq. (2). In this equation $F(x)=-f(x)$ is the dimensionless restoring force and $f(x)$ is a nonlinear function. If $f(x)$ does not have For small $x$, if $f(x)$ does not have a dominant term proportional to $x$, then Eq. (15) is said to be a "truly nonlinear oscillator" [24] Eq. (15) corresponds to a conservative system and has the "potential function"

$$
V(x)=\int f(x) \mathrm{d} x
$$

This system oscillates with an unknown frequency $\omega$ (thus a period $T=2 \pi / \omega)$.

If we compare Eq. (15) with Eq. (1), we conclude that Eq. (14) corresponds to a nonlinear oscillatory system for which there is no linear term and no perturbation parameter exists. In Eq. (14) the nonlinear function $f(x)$ can not be treated as a perturbation and thus it is evident that the alternative Lindstedt-Poincare perturbation procedure summarized in section 2 cannot be applied to Eq. (15). 
However, we will show that with some "small" modifications it is possible to apply the alternative Lindstedt-Poincaré method to Eq. (15). We will also show that, unlike the standard perturbation methods, this modified alternative Lindstedt-Poincaré perturbation procedure is applicable to truly nonlinear oscillatory systems (without a linear term). This modified alternative Lindstedt-Poincaré approach was developed by He and is known as "homotopy perturbation method" [23].

To apply the alternative Lindsted-Poincaré method, a linear term and a perturbation parameter are necessary so we need to introduce them in Eq. (15). Firstly, it is possible to introduce a linear term by rewriting Eq. (15) as follows

$$
\frac{\mathrm{d}^{2} x(t)}{\mathrm{d} t^{2}}+x(t)=x(t)-f(x(t))
$$

and secondly, a "perturbation parameter" $\lambda$ can be introduced in the following manner

$$
\frac{\mathrm{d}^{2} x(t)}{\mathrm{d} t^{2}}+x(t)=\lambda[x(t)-f(x(t))]
$$

When we set $\lambda$ equal to one we recover the original equation (Eq. (15)) that does not contain a term proportional to $x$. Now we use the parameter $\lambda$ to expand the solution $x(t)$ and the square of the unknown angular frequency $\omega$ as follows

$$
\begin{gathered}
x(t)=x_{0}(t)+\lambda x_{1}(t)+\lambda^{2} x_{2}(t)+\ldots \\
1=\omega^{2}-\lambda \alpha_{1}-\lambda^{2} \alpha_{2}-\ldots
\end{gathered}
$$

Substituting Eqs. (19) and (20) into Eq. (18) gives

$$
\begin{gathered}
\left(x_{0}^{\prime \prime}+\lambda x_{1}^{\prime \prime}+\lambda^{2} x_{2}^{\prime \prime}+\ldots\right)+\left(\omega^{2}-\lambda \alpha_{1}-\lambda^{2} \alpha_{2}-\ldots\right)\left(x_{0}+\lambda x_{1}+\lambda^{2} x_{2}+\ldots\right) \\
=\lambda\left[\left(x_{0}+\lambda x_{1}+\lambda^{2} x_{2}+\ldots\right)-f\left(\left(x_{0}+\lambda x_{1}+\lambda^{2} x_{2}+\ldots\right)\right)\right]
\end{gathered}
$$


and equating the terms with the identical powers of $\lambda$, we can obtain a series of linear equations, of which we write only the first four

$$
\begin{aligned}
& x_{0}^{\prime \prime}+\omega^{2} x_{0}=0 \\
& x_{1}^{\prime \prime}+\omega^{2} x_{1}=\left(1+\alpha_{1}\right) x_{0}-f\left(x_{0}\right) \\
& x_{2}^{\prime \prime}+\omega^{2} x_{2}=\alpha_{2} x_{0}+\left(1+\alpha_{1}\right) x_{1}-x_{1} f^{\prime}\left(x_{0}\right) \\
& x_{3}^{\prime \prime}+\omega^{2} x_{3}=\alpha_{3} x_{0}+\alpha_{2} x_{1}+\left(1+\alpha_{1}\right) x_{2}-3 x_{2} f^{\prime}\left(x_{0}\right)-\frac{1}{2} x_{1}^{2} f^{\prime \prime}\left(x_{0}\right)
\end{aligned}
$$

The initial conditions are replaced by

$$
x_{0}(0)=A, \quad x_{0}^{\prime}(0)=0, \quad x_{j}(0)=0, \quad x_{j}^{\prime}(0)=0, \quad j \geq 1
$$

In these equations we have taken into account an expression for $f\left(\sum_{n=0}^{\infty} \lambda^{2} x_{n}(t)\right)$ similar to Eq. (14). In Eqs. (23) and (25) we choose the value of the coefficients $\alpha_{n}$ in order to remove any secular term from the perturbation equation of order $n$.

\section{The cubic oscillator}

The cubic oscillator is a truly nonlinear oscillator. This is the simplest symmetrical truly nonlinear oscillator and its potential is

$$
V(x)=\frac{1}{4} \beta x^{4}, \quad \beta>0
$$

For this oscillator, the dimensionless restoring force $f(x)$ is given by the following equation

$$
f(x)=-\beta x^{3}, \quad \beta>0
$$


Substituting Eq. (28) into Eq. (15) gives the following equation of motion of a nonlinear oscillator

$$
\frac{\mathrm{d}^{2} x(t)}{\mathrm{d} t^{2}}+\beta x^{3}=0, \quad \beta>0
$$

with initial conditions $x(0)=A$ and $x^{\prime}(0)=0$. Eq. (29) corresponds to a mechanical oscillator for which the restoring force is proportional to the cube of the displacement. As we can see the linear term, $x$, is omitted in the equation and for all values of $\beta$ the motion is always nonlinear. It is clear that none of the standard methods for constructing a perturbation solution to this equation apply since these procedures assume that when $\beta$ $=0$ the resulting differential equation is that for the harmonic oscillator [24]. One example of this nonlinear oscillator is a cube-law air track oscillator [38]. The motion of a ball-bearing oscillating in a glass tube that is bent into a curve [23] is another example, as well as the motion of a mass attached to two identical stretched elastic wires for small amplitudes when the length of each wire without tension is the same as half the distance between the ends of the wires [39].

The alternative Lindstedt-Poincaré method does not work for this example. However, we can apply the homotopy perturbation method considered in section 3. Eq. (29) can be rewritten as follows

$$
\frac{\mathrm{d}^{2} x(t)}{\mathrm{d} t^{2}}+x=\lambda\left(x-\beta x^{3}\right), \quad \beta>0
$$

Taking into account Eqs. (19)-(26), we have the following linear differential equations

$$
\begin{aligned}
& x_{0}^{\prime \prime}+\omega^{2} x_{0}=0 \\
& x_{1}^{\prime \prime}+\omega^{2} x_{1}=\left(1+\alpha_{1}\right) x_{0}-\beta x_{0}^{3} \\
& x_{2}^{\prime \prime}+\omega^{2} x_{2}=\alpha_{2} x_{0}+\left(1+\alpha_{1}\right) x_{1}-3 \beta x_{1} x_{0}^{2} \\
& x_{3}^{\prime \prime}+\omega^{2} x_{3}=\alpha_{3} x_{0}+\alpha_{2} x_{1}+\left(1+\alpha_{1}\right) x_{2}-3 \beta x_{2} x_{0}^{3}-3 \beta x_{1}^{2} x_{0}
\end{aligned}
$$


The solution of Eq. (31) is

$$
x_{0}(t)=A \cos \omega t
$$

Substitution of Eq. (35) into Eq. (32) gives

$$
x_{1}^{\prime \prime}+\omega^{2} x_{1}=\left(1+\alpha_{1}-\frac{3}{4} \beta A^{2}\right) A \cos \omega t-\frac{1}{4} \beta A^{3} \cos 3 \omega t
$$

No secular terms in $x_{1}(t)$ requires that

$$
\alpha_{1}=-1+\frac{3}{4} \beta A^{2}
$$

and the solution of Eq. (36) is

$$
x_{1}(t)=-\frac{1}{32 \omega^{2}} \beta A^{3}(\cos \omega t-\cos 3 \omega t)
$$

The existence of computer algebra software, such as MATHEMATICA, allows the procedure to be extended to higher orders.

Proceeding exactly in the same way at the second order it is possible to obtain

$$
\alpha_{2}=-\frac{3}{128 \omega^{2}} \beta^{2} A^{4}, \quad x_{2}(t)=-\frac{1}{1024 \omega^{4}} \beta^{2} A^{5}(\cos \omega t-\cos 5 \omega t)
$$

and for the third order

$$
\alpha_{3}=0, \quad x_{3}(t)=-\frac{1}{32768 \omega^{6}} \beta^{3} A^{7}(7 \cos \omega t-6 \cos 5 \omega t-\cos 7 \omega t)
$$

Therefore, taking into account Eqs. (37) and (40), it is possible to obtain the approximate frequency, the period and the solution for each approximation. 
Therefore, the first approximation to the periodic solution is given by

$$
\begin{gathered}
\omega_{a 1}(A)=\frac{\sqrt{3}}{2} \sqrt{\beta} A=0.866025 \sqrt{\beta} A \\
T_{a 1}(A)=\frac{4 \pi}{\sqrt{3} \sqrt{\beta} A}=\frac{7.25520}{\sqrt{\beta} A} \\
x_{a 1}(t)=\frac{23}{24} A \cos \omega_{a 1} t+\frac{1}{24} A \cos 3 \omega_{a 1} t=0.958333 A \cos \omega_{a 1} t+0.041666 A \cos 3 \omega_{a 1} t
\end{gathered}
$$

For the second approximation we have

$$
\begin{gathered}
\omega_{a 2}(A)=\frac{1}{4} \sqrt{6+\sqrt{30}} \sqrt{\beta} A=0.846951 \sqrt{\beta} A \\
T_{a 2}(A)=\frac{8 \pi}{\sqrt{6+\sqrt{30}} \sqrt{\beta} A}=\frac{7.41859}{\sqrt{\beta} A} \\
x_{a 2}(t)=\frac{A}{4(6+\sqrt{30})^{2}}\left[(251+46 \sqrt{30}) \cos \omega_{a 2} t+2(6+\sqrt{30}) \cos 3 \omega_{a 2} t+\cos 5 \omega_{a 2} t\right] \\
=0.954538 A \cos \omega_{a 2} t+0.043564 A \cos 3 \omega_{a 2} t+0.0018979 A \cos 5 \omega_{a 2} t
\end{gathered}
$$

And for the third approximation we have

$$
\begin{gathered}
\omega_{a 3}(A)=\omega_{a 2}(A)=\frac{1}{4} \sqrt{6+\sqrt{30}} \sqrt{\beta} A=0.846951 \sqrt{\beta} A \\
T_{a 3}(A)=T_{a 2}(A)=\frac{8 \pi}{\sqrt{6+\sqrt{30}} \sqrt{\beta} A}=\frac{7.41859}{\sqrt{\beta} A}
\end{gathered}
$$




$$
\begin{aligned}
x_{a 3}(t) & =0.953959 A \cos \omega_{a 2} t+0.0440606 A \cos 3 \omega_{a 2} t+0.0018979 A \cos 5 \omega_{a 2} t \\
& +0.00008268 A \cos 7 \omega_{a 2} t
\end{aligned}
$$

\section{Comparison with the exact and other approximate solutions}

We illustrate the accuracy of the modified approach by comparing the approximate solutions previously obtained with the exact period $T_{e x}$ and other results in the literature. In particular we will consider the solution of Eq. (29) by means of the harmonic balance method [15]. This method is a procedure for determining analytical approximations to the periodic solutions of differential equations using a truncated Fourier series representation [17]. Like the homotopy perturbation method, the harmonic balance method can be applied to nonlinear oscillatory problems where a linear term does not exist, the nonlinear terms are not small, and there is no perturbation parameter. However, it is very difficult to use the harmonic balance method to construct higherorder analytical approximations because this method requires solving analytical solutions of sets of algebraic equations with very complex nonlinearities [18].

By applying the first approximation based on the method of harmonic balance, Mickens achieved the following expressions for the frequency and the period [15]

$$
\begin{gathered}
\omega_{M 1}(A)=\frac{\sqrt{3}}{2} \sqrt{\beta} A=0.866025 \sqrt{\beta} A \\
T_{M 1}(A)=\frac{4 \pi}{\sqrt{3} \sqrt{\beta} A}=\frac{7.25520}{\sqrt{\beta} A}
\end{gathered}
$$

and the approximate solution

$$
x_{M 1}(t)=A \cos \omega_{M 1} t
$$


While applying the second approximation based on the method of harmonic balance, Mickens obtained [15]

$$
\begin{gathered}
\omega_{M 2}(A)=0.8507 \sqrt{\beta} A \\
T_{M 2}(A)=\frac{7.3859}{\sqrt{\beta} A} \\
x_{M 2}(t)=0.9569 A \cos \omega_{M 2} t+0.0431 A \cos 3 \omega_{M 2} t
\end{gathered}
$$

Calculation of the exact period proceeds as follows. Integrating Eq. (29) and using the initial conditions we arrive at

$$
\frac{1}{2}\left(\frac{\mathrm{d} x}{\mathrm{~d} t}\right)^{2}+\frac{1}{4} \beta x^{4}=\frac{1}{4} \beta A^{4}
$$

From the representation above, we can derive the exact period $T_{e x}$ as follows (see the Appendix)

$$
T_{e x}(A)=\frac{4}{A \sqrt{\beta}} K(1 / 2)=\frac{7.41630}{\sqrt{\beta} A}
$$

where $K(m)$ is the complete elliptical integral of the first kind [40]. The exact frequency is given by the following expression

$$
\omega_{e x}(A)=\frac{2 \pi}{4 K(1 / 2)} A \sqrt{\beta}=0.84721 \sqrt{\beta} A
$$

The exact solution to Eq. (29) is (see the Appendix) 


$$
x_{e x}(t)=\operatorname{Acn}\left(\sqrt{\beta} A t ; \frac{1}{2}\right)
$$

where $c n$ is the Jacobi elliptic function which has the following Fourier expansion [41]

$$
c n(u ; m)=\frac{2 \pi}{\sqrt{m} K(m)} \sum_{n=0}^{\infty} \frac{q^{n+1 / 2}}{1+q^{2 n+1}} \cos \left[\frac{(2 n+1) \pi u}{2 K(m)}\right]
$$

where

$$
q(m)=\exp \left\lfloor-\frac{\pi K\left(m^{\prime}\right)}{K(m)}\right\rfloor
$$

and

$$
m^{\prime}=1-m
$$

With these results, the Fourier expansion of Eq. (59) becomes

$$
x_{e x}(t)=A c n\left(\sqrt{\beta} A t ; \frac{1}{2}\right)=\frac{2 \pi \sqrt{2} A}{K(1 / 2)} \sum_{n=0}^{\infty}\left(\frac{\exp [(n+1 / 2) \pi]}{1+\exp [(2 n+1) \pi]}\right) \cos \left[(2 n+1) \omega_{e x} t\right]
$$

where we have taken into account Eq. (58) and from Eq. (61) $q(1 / 2)=\exp (-\pi)$.

The first terms of this Fourier expansion are

$$
\begin{aligned}
x_{e x}(t) & =0.95501 A \cos \omega_{e x} t+0.043050 A \cos 3 \omega_{e x} t \\
& +0.0018605 A \cos 5 \omega_{e x} t+0.0000804 A \cos 7 \omega_{e x} t+\ldots
\end{aligned}
$$

In order to compare the approximate and the exact periods we obtain the following expressions 


$$
\begin{array}{ll}
\sqrt{\beta} A T_{e x}(A)=7.41630 & \\
\sqrt{\beta} A T_{M 1}(A)=7.25520 & \text { Relative error }=2.2 \% \\
\sqrt{\beta} A T_{a 1}(A)=7.25520 & \text { Relative error }=2.2 \% \\
\sqrt{\beta} A T_{M 2}(A)=7.3859 & \text { Relative error }=0.41 \% \\
\sqrt{\beta} A T_{a 2}(A)=7.41859 & \text { Relative error }=0.031 \%
\end{array}
$$

As we can see, the approximate periods $T_{M 1}$ and $T_{a 1}$ are equal, but $T_{a 2}$ is more accurate than Mickens' period $T_{M 2}$ and can provide excellent approximations to the exact frequency for the whole range of values of the oscillation amplitude. Therefore, for any values of $\beta$, it can be easily proved that the relative error of the approximate period $T_{a 2}$ obtained in this paper is $0.031 \%$ in the whole solution domain $(0<\beta<\infty)$ and for the complete range of oscillation amplitude, including the limiting cases of amplitude approaching zero and infinity. However, for Mickens' period $T_{M 2}$ the relative error is $0.41 \%$, i.e., more than thirteen times the relative error for $T_{a 2}$.

The normalized periodic exact solution, $x_{e x}(t) / A$, achieved using Eq. (59), Mickens' first order approximate periodic solution, $x_{M 1}(t) / A$ (Eq. (52)), and the proposed first order approximate solution, $x_{a 1}(t) / A$ (Eq. (43)) are plotted in Figure 1, where $h$ is defined as follows

$$
h=\frac{A \sqrt{\beta}}{4 K(1 / 2)} t
$$

This figure is valid for all values of oscillation amplitude and for $\beta>0$. In Figure 2 we compare the normalized exact solution, $x_{e x}(t) / A$, with the approximate solutions obtained by means of the second-order harmonic balance method, $x_{M 2}(t) / A$ (Eq. (55)), and the second order approximation obtained using the homotopy perturbation method, $x_{a 2}(t) / A$ (Eq. (46)). As we can see, $x_{a 2}(t) / A$ (red line) coincides with the exact solution $x_{e x}(t) / A$ (black line). We can not see the black line because it is under the red line. Figure 2 shows that the second approximate analytical solution obtained by means of He's homotopy perturbation method provides a most excellent approximation to the exact 
periodic solution. Figures 1 and 2 also show that Eqs. (43) and (46) can provide better approximations than Eqs. (52) and (55), respectively, to the exact periodic solutions.

If we compare the approximate solutions for the first and the second approximations with the Fourier expansion of the exact solution (Eq. (64)), we can see that $x_{a 1}(t)$ is a better approximation than $x_{M 1}(t)$, although the periods for this approximation are equal. For the second approximation the approximate solution $x_{a 2}(t)$ is a better approximation than $x_{M 2}(t)$ and the approximate period obtained using the second order homotopy perturbation method is also better than the period obtained by means of the second order harmonic balance method. Obviously, $x_{a 3}(t)$ is the best approximation. If we compare $x_{a 3}(t)$ (Eq. (49)) with the first four terms of the Fourier expansion of $x_{e x}(t)$ (Eq. (64)) we can see that the relative errors for these terms are $0.1 \%$, $0.02 \%, 0.02 \%$ and $0.03 \%$, respectively.

Finally, we compare the values of the solution when the value of the time $t$ is equal to one exact period. For $t=T_{e x}$ we have $x_{e x}\left(T_{e x}\right)=A$ and the results for the different analytical approximate solutions are

$$
\begin{array}{ll}
x_{M 1}\left(T_{e x}\right)=0.990283 A & \text { Relative error }=1.0 \% \\
x_{a 1}\left(T_{e x}\right)=0.991848 A & \text { Relative error }=0.8 \% \\
x_{M 2}\left(T_{e x}\right)=0.999666 A & \text { Relative error }=0.03 \% \\
x_{a 2}\left(T_{e x}\right)=0.999998 A & \text { Relative error }=0.0002 \%
\end{array}
$$

These results are an indication of the accuracy of the homotopy perturbation method as applied to this particular problem and show that it provides an excellent approximation to the solution of Eq. (29).

\section{Conclusions}

The homotopy perturbation method has been applied to obtain analytical approximate solutions for truly nonlinear problems that are conservative and periodic. The major 
conclusion is that this perturbation scheme provides excellent approximations to the solution of these nonlinear systems with high accuracy and, in particular, the results for the cubic oscillator are valid for the whole solution domain. The analytical representations obtained using the homotopy perturbation technique give excellent approximations to the exact solutions for the whole range of values of oscillation amplitude. These approximate solutions are better than the approximate solutions obtained using the harmonic balance method. For the second order approximation, the relative error of the analytical approximate frequency obtained using the homotopy perturbation approach for the cubic oscillator is $0.031 \%$, while the relative error is $0.41 \%$ when the harmonic balance method's second order approximation is considered. An interesting feature considered in this paper is the comparison between the analytical approximate solutions and the Fourier series expansion of the exact solution. This has allowed us to compare the coefficients for the different harmonics. In summary, He's homotopy perturbation method is very simple in its principle, and it can be used to solve other conservative truly nonlinear oscillators with complex nonlinearities.

\section{Appendix. Exact solution for the cubic oscillator}

Consider the differential equation with single-term positive-power nonlinearity

$$
\frac{\mathrm{d}^{2} x(t)}{\mathrm{d} t^{2}}+\beta x^{3}=0, \quad \beta>0
$$

with initial conditions

$$
x(0)=A \quad \text { and } \quad \frac{\mathrm{d} x(0)}{\mathrm{d} t}=A
$$

Integration of Eq. (1) gives the first integral

$$
\frac{1}{2}\left(\frac{\mathrm{d} x}{\mathrm{~d} t}\right)^{2}+\frac{1}{4} \beta x^{4}=\frac{1}{4} \beta A^{4}
$$


where the integration constant was evaluated using the initial conditions of Eq. (A2). Since Eq. (A1) has the first-integral in Eq. (A3), it can be concluded that all the solutions are periodic [5].

Solving Eq. (A3) for $t$ gives

$$
t(x)=-\sqrt{\frac{2}{\beta}} \int_{A}^{x} \frac{\mathrm{d} x}{\sqrt{A^{4}-x^{4}}}
$$

The linear transform $x=A u$ reduces this equation to the form

$$
t(x)=\frac{1}{A} \sqrt{\frac{2}{\beta}} \int_{x / A}^{1} \frac{\mathrm{d} x}{\sqrt{\left(1-u^{2}\right)\left(1+u^{2}\right)}}
$$

The integral in Eq. (A5) can be written in terms of elliptical integrals as follows [40]

$$
\int_{x / A}^{1} \frac{\mathrm{d} x}{\sqrt{\left(1-u^{2}\right)\left(1+u^{2}\right)}}=\int_{0}^{1} \frac{\mathrm{d} x}{\sqrt{\left(1-u^{2}\right)\left(1+u^{2}\right)}}-\int_{0}^{x / A} \frac{\mathrm{d} x}{\sqrt{\left(1-u^{2}\right)\left(1+u^{2}\right)}}
$$

The first integral is

$$
\int_{0}^{1} \frac{\mathrm{d} x}{\sqrt{\left(1-u^{2}\right)\left(1+u^{2}\right)}}=K(-1)=\frac{1}{\sqrt{2}} K(1 / 2)
$$

and the second integral can be expressed as follows [40]

$$
\begin{gathered}
\left.\int_{0}^{x / A} \frac{\mathrm{d} x}{\sqrt{\left(1-u^{2}\right)\left(1+u^{2}\right)}}=K(-1)-F(\arcsin (x / A) ;-1)\right) \\
\quad=\frac{1}{\sqrt{2}} K(1 / 2)-\frac{1}{\sqrt{2}} F\left(\frac{\pi}{2}-\arcsin (x / A) ; 1 / 2\right)
\end{gathered}
$$




$$
=\frac{1}{\sqrt{2}} K(1 / 2)-\frac{1}{\sqrt{2}} F(\arccos (x / A) ; 1 / 2)
$$

where $K(m)$ and $F(\varphi ; m)$ are the complete and the incomplete elliptical integrals of the first kind defined as follows [40]

$$
\begin{gathered}
K(m)=\int_{0}^{1} \frac{\mathrm{d} x}{\sqrt{\left(1-u^{2}\right)\left(1-m u^{2}\right)}} \\
F(\varphi ; m)=\int_{0}^{z} \frac{\mathrm{d} x}{\sqrt{\left(1-u^{2}\right)\left(1-m u^{2}\right)}}, \quad z=\sin \varphi
\end{gathered}
$$

In Eqs. (A7) and (A8) we considered the following relations [40]

$$
\begin{gathered}
K(-m)=\frac{1}{\sqrt{1+m}} K\left(\frac{m}{1+m}\right) \\
F(\varphi ;-m)=\frac{1}{\sqrt{1+m}} K\left(\frac{m}{1+m}\right)-F\left(\frac{\pi}{2}-\varphi ; \frac{m}{1+m}\right)
\end{gathered}
$$

Eqs. (A6)-(A8) allow us to write Eq. (A5) as follows

$$
t(x)=\frac{1}{\sqrt{\beta} A} F(\arccos (x / A) ; 1 / 2)
$$

This equation can be inverted to give $x$ as a function of $t$. Doing this gives

$$
x(t)=\operatorname{Acn} F(\sqrt{\beta} A t ; 1 / 2)
$$


where $c n(z ; m)$ is the Jacobi elliptic function [41].

The period of the oscillations of the cubic truly nonlinear oscillator can be obtained from Eq. (A13) as follows

$$
T=4 t(0)=\frac{4}{\sqrt{\beta} A} K(1 / 2)
$$

\section{Acknowledgements}

This work was supported by the "Ministerio de Educación y Ciencia", Spain, under project FIS2005-05881-C02-02, and by the "Generalitat Valenciana", Spain, under project ACOMP06/007. 


\section{References}

[1] D. K. Campbell, Nonlinear science: the next decade (MIT Press, Massachusetts 1992).

[2] S. Liao, Beyond Perturbation: introduction to the homotopy analysis method (CRC Press, Boca Raton FL, 2004).

[3] J. H. He, "A review on some new recently developed nonlinear analytical techniques", Int. J. Non-linear Sci. Numer. Simulation 1, 51-70 (2000).

[4] A. H. Nayfeh, Problems in Perturbations (Wiley, New York 1985).

[5] R. E. Mickens, Oscillations in Planar Dynamics Systems (World Scientific, Singapore 1996).

[6] J. H. He, "A new perturbation technique which is also valid for large parameters", J. Sound Vib. 229, 1257-1263 (2000).

[7] J. H. He, "Modified Lindstedt-Poincare methods for some non-linear oscillations. Part III: double series expansion", Int. J. Non-linear Sci. Numer. Simulation 2, 317-320 (2001).

[8] J. H. He, "Modified Lindstedt-Poincare methods for some non-linear oscillations. Part I: expansion of a constant”, Int. J. Non-linear Mech. 37, 309-314 (2002).

[9] J. H. He, "Modified Lindstedt-Poincare methods for some non-linear oscillations. Part II: a new transformation”, Int. J. Non-linear Mech. 37, 315-320 (2002). 
[10] P. Amore and A. Aranda, "Improved Lindstedt-Poincaré method for the solution of nonlinear problems", J. Sound. Vib. 283, 1115-1136 (2005).

[11] P. Amore and F. M. Fernández, "Exact and approximate expressions for the period of anharmonic oscillators", Eur. J. Phys. 26, 589-601 (2005).

[12] J. H. He, "Homotopy perturbation method for bifurcation on nonlinear problems", Int. J. Non-linear Sci. Numer. Simulation. 6, 207-208 (2005).

[13] P. Amore, A. Raya and F. M. Fernández, “Alternative perturbation approaches in classical mechanics", Eur. J. Phys. 26, 1057-1063 (2005).

[14] P. Amore. A. Raya and F. M. Fernández, "Comparison of alternative improved perturbative methods for nonlinear oscillations", Phys. Lett. A 340, 201-208 (2005).

[15] R. E. Mickens, "Comments on the method of harmonic-balance", J. Sound. Vib. 94, 456-460 (1984).

[16] R. E. Mickens, "Mathematical and numerical study of the Duffing-harmonic oscillator", J. Sound Vib. 244, 563-567 (2001).

[17] B. S. Wu and C. W. Lim, "Large amplitude nonlinear oscillations of a general conservative system”, Int. J. Non-linear Mech. 39, 859-870 (2004).

[18] C. W. Lim and B. S. Wu, "Accurate higher-order approximations to frequencies of nonlinear oscillators with fractional powers", J. Sound Vib. 281, 1157-1162 (2005). 
[19] A. Beléndez, A. Hernández, A. Márquez, T. Beléndez and C. Neipp, “Analytical approximations for the period of a simple pendulum", Eur. J. Phys. 27, 539-551 (2006)

[20] C. W. Lim and B. S. Wu, "A new analytical approach to the Duffing-harmonic oscillator", Phys. Lett. A 311, 365-373 (2003).

[21] H. Hu and J. H. Tang, "Solution of a Duffing-harmonic oscillator by the method of harmonic balance", J. Sound Vib. 294, 637-639 (2006).

[22] H. Hu, "Solution of a Duffing-harmonic oscillator by an iteration procedure", $J$. Sound Vib. 298, 446-452 (2006).

[23] J. H. He, "Some asymptotic methods for strongly nonlinear equations", Int. J. Mod. Phys. B, 20, 1141-1199 (2006).

[24] R. E. Mickens, "A generalized iteration procedure for calculating approximations to periodic solutions of truly nonlinear oscillators", J. Sound Vib. 287, 1045-1051 (2005)

[25] J. H. He, "New interpretation of homotopy perturbation method", Int. J. Mod. Phys. B, 20, 2561-2568 (2006).

[26] J. H. He, "Homotopy perturbation method for bifurcation of nonlinear problems", Int. J. Non-linear Sci. Numer. Simulation 6 (2), 207-208 (2005).

[27] X. C. Cai, W. Y. Wu and M. S. Li, "Approximate period solution for a kind of nonlinear oscillator by He's perturbation method", Int. J. Non-linear Sci. Numer. Simulation 7 (1), 109-70 (2006). 
[29] S. Abbasbandy, “Application of He's homotopy perturbation method for Laplace transform", Chaos, Solitons \& Fractals 30, 1206-1212 (2006).

[30] S. Abbasbandy, "The application of homotopy analysis method to nonlinear equations arising in heat transfer", Phys. Lett. A (in press).

[31] D. D. Ganji, “The application of He's homotopy perturbation method to nonlinear equations arising in heat transfer", Phys. Lett. A 355, 337-341 (2006).

[32] P. D. Ariel, T. Hayat and S. Asghar, "Homotopy perturbation method and axisymmetric flow over a stretching sheet", Int. J. Non-linear Sci. Numer. Simulation 7 (4), 399-406 (2006).

[34] D. D. Ganji and A. Sadighi, “Application of He's homotopy-perturbation method to nonlinear coupled systems of reaction-diffusion equations", Int. J. Non-linear Sci. Numer. Simulation 7 (4), 411-418 (2006).

[35] J. H. He, "Application of homotopy perturbation method to nonlinear wave equations", Chaos, Solitons \& Fractals 36, 695-700 (2005).

[36] M. El-Shaded, “Application of He's homotopy perturbation method to Volterra's integro-differential equation", Int. J. Non-linear Sci. Numer. Simulation. 6 (2), 163-168 (2005).

[37] A. Siddiqui, R. Mahmood and Q. Ghori, "Thin film flow of a third grade fluid on moving a belt by He's homotopy perturbation method", Int. J. Non-linear Sci. Numer. Simulation. 7 (1), 15-26 (2006).

[38] S. Whineray, “A cube-law air track oscillator”, Eur. J. Phys. 12, 90-95 (1991). 
[39] J. B. Marion, Classical Dynamics of Particles and Systems (Harcourt Brace Jovanovich, San Diego, CA 1970)

[40] L. M. Milne-Thomson, "Elliptic integrals" in M. Abramowitz and I. A. Stegun (Eds.), Handbook of Mathematical Functions, Dover Publications, Inc., New York, 1972.

[41] L. M. Milne-Thomson, "Jacobi elliptic functions and theta functions" in M. Abramowitz and I. A. Stegun (Eds.), Handbook of Mathematical Functions, Dover Publications, Inc., New York, 1972. 


\section{FIGURE CAPTIONS}

Figure 1.- Comparison of first order approximate solutions obtained using the homotopy perturbation (red line) and the harmonic balance method (blue line) with the exact solution (black line).

Figure 2.- Comparison of second order approximate solutions obtained using the homotopy perturbation (red line) and the harmonic balance method (blue line) with the exact solution (black line). The black line can not be seen because it is under the red line. 


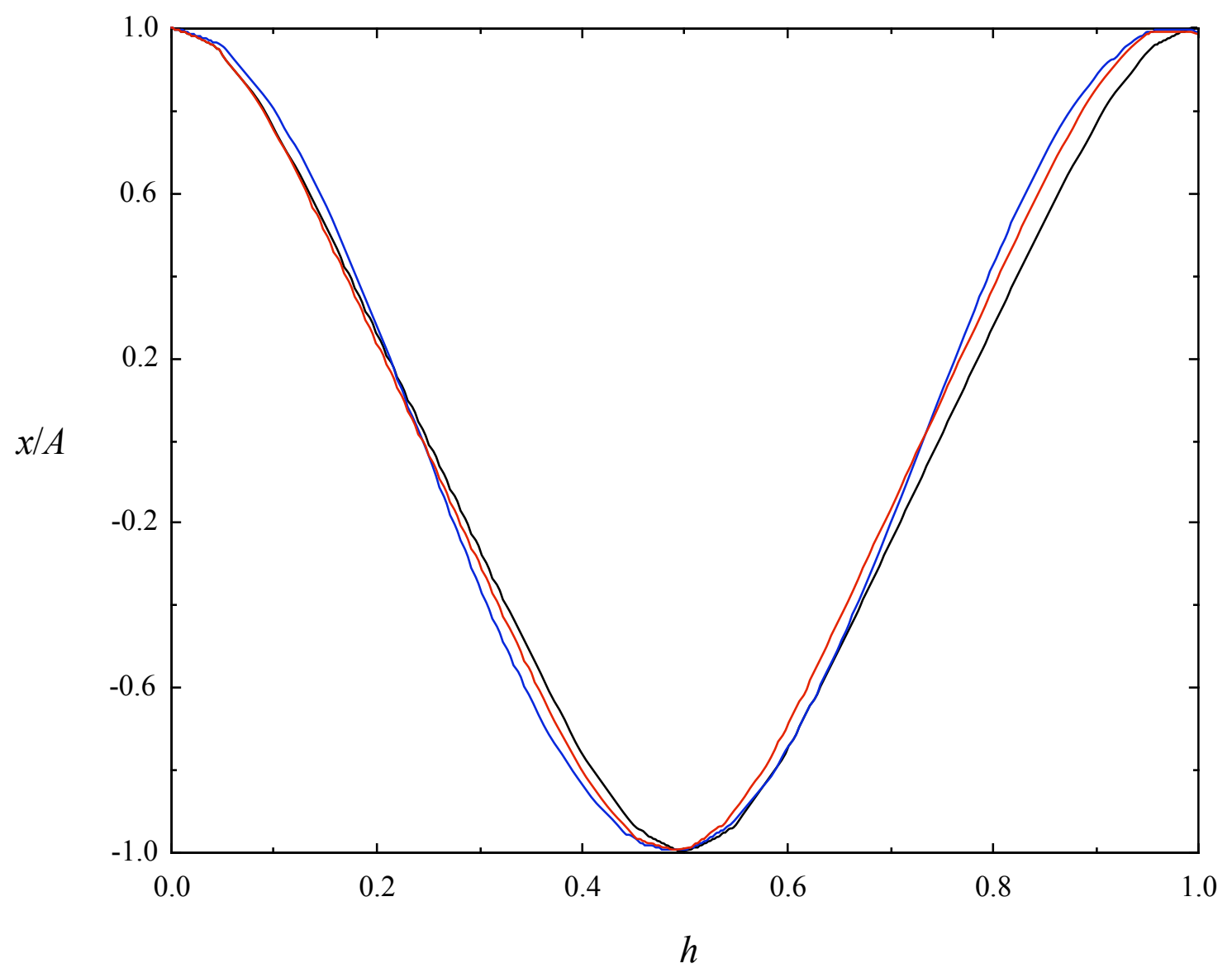

FIGURE 1 


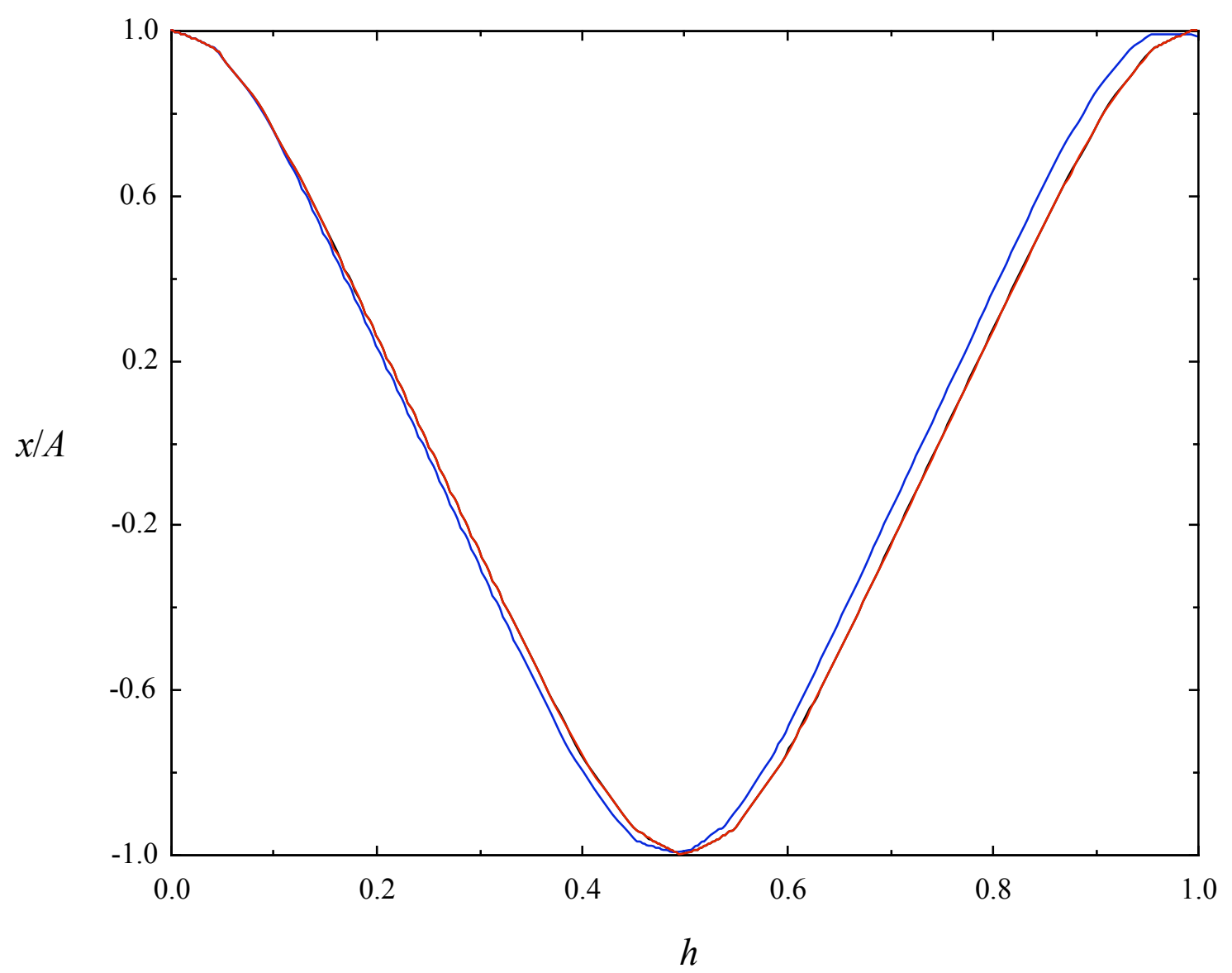

FIGURE 2 